\title{
A $G$-SIGNATURE THEOREM FOR $V$-MANIFOLDS
}

\author{
CHAO-CHU LIANG
}

\begin{abstract}
We prove an analogue of the Atiyah-Singer $G$-signature theorem for an isometry $f$ on a $V$-manifold.
\end{abstract}

An $n$-dimensional $V$-manifold is a space locally homeomorphic to the orbit space of an orthogonal action on the Euclidean space $R^{n}$ by a finite group. Kawasaki [7] proved the analogue of the Hirzebruch signature theorem for $V$-manifolds. In this paper we will show that the argument in [7] can be modified slightly to give us an analogue of the Atiyah-Singer $G$-signature theorem [3] for an isometry $f$ on a $V$-manifold.

1. We recall here some definitions concerning $C^{\infty} V$-manifolds, and refer the readers to [4], [8] and [9] for details.

Let $G_{U}$ be a finite group acting linearly on $R^{n}$ with the fixed point set of dimension at most $n-2, U^{\sharp}$ an invariant open subset of $R^{n}$ and $q_{U}$ : $U^{\sharp} \rightarrow U=U^{\sharp} / G_{U}$ the projection map satisfying $q_{U} \circ g=q_{U}$ for all $g \in G_{U}$. Then we call $U$ a local $V$-manifold. A $V$-map $f$ from one $V$-manifold $U$ to another $W$ is a $\left(G_{U}, G_{W}\right)$-equivariant map $f^{\sharp}$ from $U^{\sharp}$ to $W^{\sharp}$; that is, there exists a homomorphism $\lambda: G_{U} \rightarrow G_{W}$ such that $\lambda(g) \circ f^{\sharp}=f^{\sharp} \circ g$ for $g \in G_{U}$.

A $V$-manifold structure on a Hausdorff paracompact space $X$ consists of an open covering $X$ by local $V$-manifolds $\{U\}$ such that the overlap maps are $V$-diffeomorphisms. Each $\left\{U^{\sharp}, G_{U}\right\}$ (or $\left\{U^{\sharp}, G_{U}, q_{U}\right\}$ ) is called a local uniformizing system (1.u.s.) for $U$ and $G_{U}$ a local group [9]. A $C^{\infty}$-map is given by a compatible family of equivariant maps on l.u.s.'s. We also have the notion of $V$-bundle map [9]. In particular, using the differentials of the elements in $G_{U}$, we may construct the tangent $V$-bundle $T X$.

From now on, we assume that all the $V$-manifolds are orientable, that is, all the elements of $G_{U}$ and the overlap maps preserve the orientations.

A Riemannian metric can be introduced on $X$, given locally by a $G_{U}$-invariant metric on $U^{\sharp}[4$, p. 864]. The Chern-Weil construction of the characteristic classes also works in this category [7].

Let $w$ be an $n$-form on $X$. Then we define the integral $\int_{X} w$ via a partition of unity $\left\{\varphi_{U}\right\}$ as follows:

$$
\int_{X} w=\sum_{\{U\}}\left|G_{U}\right|^{-1} \int_{U^{\sharp}} \varphi_{U}^{\sharp} w_{U}^{\#}
$$

Received by the editors July 7, 1978 and, in revised form, August 31, 1978.

AMS (MOS) subject classifications (1970). Primary 58G10.

Key words and phrases. $G$-signature, $V$-manifold, elliptic operators.

${ }^{1}$ Supported by the University of Kansas General Research Fund. 
(for an object on $U$, we use the superscript \# to denote its corresponding object on $U^{\sharp}$ ).

If $p^{\sharp} \in U^{\sharp}$ satisfying $q_{U}\left(p^{\sharp}\right)=p$, then the isotropy subgroup of $G_{U}$ at $p^{\sharp}$ depends only on $p$, and is denoted by $G_{p}$ if no confusion will arise [9]. A point $p$ with $G_{p}=\{1\}$ is called a regular point, otherwise a singular point. Let $\Sigma X$ denote the set of all singular points. According to [7, p. 77], we may express $\Sigma X$ as a union of $V$-manifolds of lower dimensions as follows: Let $U_{p}$ be a neighborhood of $p$ with $p^{\sharp}$ as the origin of $U_{p}^{\sharp}$, and $(1)=\left(h_{p}^{0}\right), \ldots,\left(h_{p}^{r_{p}}\right)$ be all the conjugacy classes of $G_{p}$. Also, let $Z_{G_{p}}\left(h_{p}^{j}\right)$ denote the centralizer of $h_{p}^{j}$ in $G_{p},\left(U_{p}^{\sharp}\right)^{h_{j}^{j}}$ the set of all elements of $U_{p}^{\sharp}$ fixed by $h_{p}^{j}$ and $K_{p}^{j}$ the kernel of the representation $Z_{G_{p}}\left(h_{p}^{j}\right) \rightarrow G L\left(\left(U_{p}^{\sharp}\right)^{h^{j}}\right)$. Then the $V$-manifold (of various dimensions) $F X$ is given locally by $F U_{p}=\amalg_{j=1}^{r_{p}}\left(U_{p}^{\#}\right)^{h j} / Z_{G_{p}}\left(h_{p}^{j}\right)$ (disjoint unions) with l.u.s. $\left\{\left(U_{p}^{\sharp}\right)^{h t}, Z_{G_{p}}\left(h_{p}^{j}\right) / K_{p}^{j}\right\}$. Each connected component $F X_{\lambda}$ of $F X$ is a $V$-manifold, and is assigned a number $m_{\lambda}=\left|K_{p}^{\lambda}\right|$, the order of $K_{p}^{\lambda}$.

2. Let $E$ denote a vector $V$-bundle over a compact $V$-manifold $X$. The set $\Gamma(X, E)$ (or just $\Gamma(E)$ ) consists of all the $C^{\infty}$-sections $h: X \rightarrow E$ defined locally by equivariant sections $h_{U}^{\#}: U^{\#} \rightarrow E_{U}^{\#}$. We write $E^{\prime}$ for the dual of $E$, and $\Lambda^{k}$ the $k$ th exterior power of the cotangent bundle $T^{*} X$.

Defined locally as $G_{U}$-equivariant objects over each l.u.s. $U^{\sharp}$, we also have the notions of pseudo-differential operators, symbols, ellipticity, parametrices, and index for an elliptic operator, etc. (see [6] or [7]).

We describe next the kernel of a smoothing operator [6, p. 80], [7, p. 82] $A$ : $\Gamma(X, E) \rightarrow \Gamma(X, F)$. Suppose that locally $K_{U}^{\sharp}(x, y) \in \Gamma\left(U^{\sharp} \times U^{\sharp}, F_{U}^{\#} \times\left(E_{U}^{\prime \#}\right.\right.$ $\left.\otimes \Lambda^{n}\right)$ ) is a $C^{\infty}$-kernel of the corresponding $G_{U}$-invariant operator $A_{U}^{\#}$ : $\Gamma\left(U^{\sharp}, E_{U}^{\sharp}\right) \rightarrow \Gamma\left(U^{\sharp}, F_{U}^{\sharp}\right)$. We have $K_{U}^{\sharp}(x, y)=\left(g^{-1}, g^{-1}\right) K_{U}^{\sharp}(g x, g y)$. But $K_{U}^{\#}$ is not $G_{U} \times G_{U}$-invariant, hence does not define a section over $U \times U$. Thus locally, the kernel for $A_{U}$ is defined to be

$$
K_{U}(x, y)=\sum_{g \in G_{U}}\left(g^{-1}, 1\right) K_{U}^{\sharp}(g x, y) .
$$

3. Let $f$ be an orientation-preserving isometry of a compact orientable $V$-manifold $X$, that is, $f: X \rightarrow X$ is given locally as a local equivariant isometry $f^{\sharp}$ from one l.u.s. to another $\left(G_{U}\right.$ acts as an isometry on each l.u.s. $U^{\sharp)}$.

We assume that (I) the order of $f$ is relatively prime to the order of the elements in each local group $G_{U}$, and none of the orders is even; and (II) the fixed point set of $f$ is an oriented $V$-submanifold $Y$ in $X$, and $Y_{i}=Y \cap F X_{i}$ is a $V$-submanifold of $F X_{i}$ for each $i$ (this follows from the first part of (II) and (I)).

If $X$ is of even dimension, then we may define the $G$-signature $\operatorname{sign}(f, X)$ as in [3, pp. 578-579]. Also, we may apply the Hodge star operator * [4] to the de Rham complex $\left(\Lambda^{i} \otimes \mathbf{C}, d\right)[8]$ to construct the signature complex $0 \rightarrow \Lambda^{+}$ $\stackrel{D}{\rightarrow} \Lambda^{-} \rightarrow 0$ as in [3]. The same algebraic argument in [3, pp. 578-579] gives us the formula $\operatorname{sign}(f, X)=\operatorname{index}(D)(f)$. 
We write $f$ for $f^{\sharp}$ on each l.u.s. if no confusion will arise. Since the order of $f$ is relatively prime to the order of $g \in G_{U}$, we see that $f g x=x \Rightarrow g f x=x \Rightarrow$ $f x=g^{-1} x \Rightarrow g x=x$ and $f x=x$. Therefore, locally we have $\operatorname{Fix}(f g)=$ $\operatorname{Fix}(f) \cap \operatorname{Fix}(g)$.

Recall that locally $F X_{j}=\operatorname{Fix}(g)$ for some $g \in G_{U}$. Hence $Y_{j}^{\#}=\operatorname{Fix}(f g)$ locally. We let $N_{j}=N\left(Y_{j}^{\sharp}\right)=N($ Fix $(f g))$ denote the normal bundle of $Y_{j}^{\sharp}=$ $\operatorname{Fix}(f g)$ in $U^{\sharp}$. Then $N_{j}$ splits into eigenspaces of $f g: N_{j}=N_{\theta_{1}} \oplus \cdots \oplus N_{\theta_{s}}$, ( $f g) v=e^{i \theta} \cdot v$ if $v \in N_{\theta}$. These splittings are preserved by the action of $Z_{G_{v}}(g)$ and the attaching map of $N_{j}$. Hence we have a global splitting, denoted also by $N_{j}=N_{\theta_{1}} \oplus \cdots \oplus N_{\theta_{s}}$, for each $j=1,2, \ldots, c$. Since the order of $f g$ is odd, we have Chern classes $c\left(N_{\theta}\right)=\Pi_{k=1}^{\nu} \operatorname{coth}\left(x_{k}+i \theta / 2\right)$.

We may also define the $L$-class of a real vector $V$-bundle by the $L$-polynomial in its Pontrjagin classes. Let $L^{Y_{j}}=L\left(T Y_{j}\right) \Pi_{k=1}^{s} L_{\theta_{k}}\left(N_{\theta_{k}}\right)$.

THEOREM. Let $f$ be an orientation-preserving isometry on a compact orientable $V$-manifold $X^{2 n}$ with $\operatorname{Fix}(f)=Y$ satisfying the conditions (I) and (II) above. Then we have

$$
\operatorname{Sign}(f, X)=\left\langle L^{Y},[Y]\right\rangle+\sum_{j=1}^{c} \frac{1}{m_{j}}\left\langle L^{Y_{j}},\left[Y_{j}\right]\right\rangle .
$$

Proof. The argument given in [7] can be adapted here. Let $A_{1}=D^{*} D$ and $A_{2}=D D^{*}$ denote the adjoint of $D$. Both $A_{1}$ and $A_{2}$ are self-adjoint, positive definite, elliptic operators (on vector $V$-bundles) of order 2 . Hence the spectrum of $A_{1}$ (also $A_{2}$ ) is discrete and positive [6, p. 82]. Let $\zeta_{A}(z)$ denote the zeta-function of $A$, and for $u>0, u^{z}$ ind $(D)=\zeta_{A_{1}+u}(z)-\zeta_{A_{2}+u}(z)$ as in [6] or [7]; we may take $z=0$ and let $u$ approach 0 .

Using the notation in [6, pp. 25, 84] or [7, pp. 79-82], we see that

$$
\zeta_{A}(z)(f)=\int_{X} d \operatorname{tr}\left[\left(f^{-1}, 1\right) K_{z}(f x, y)\right], \quad \operatorname{Re}(2 z)<-2 n .
$$

Let $\left\{\varphi_{U}\right\}$ be a partition of unity. We have from (2.1) that

$$
\zeta_{A}(0)(f)=\sum_{U}\left|G_{U}\right|^{-1} \sum_{g \in G_{U}} \int_{U^{\sharp}} \varphi_{U}(x) d \operatorname{tr}\left[\left(g^{-1} f^{-1}, 1\right) K_{z, U}^{\sharp}(f g x, x)\right]
$$

(see [6, p. 85] or [7]).

Then the argument in [6, p. 85] or [7] gives us

$$
\operatorname{ind}(D)(f)=\int_{Y} d\left(Z_{A_{1}}^{f}-Z_{A_{2}}^{f}\right)+\sum_{j=1}^{c} \frac{1}{m_{j}} \int_{Y_{j}} d\left(Z_{A_{1}}^{Y_{j}}-Z_{A_{2}}^{Y_{j}}\right),
$$

where locally $d Z_{A_{i}}^{Y_{j}}=d Z_{A_{i}}^{f_{g}}$, which is defined in [7, p. 80].

By resorting to the local version of the Atiyah-Singer $G$-signature theorem as in [7] or [2], we may identify the $j$ th term on the right-hand side of the equality above with $\int_{Y_{j}} L^{Y_{j}}=\left\langle L^{Y_{j}},\left[Y_{j}\right]\right\rangle$. Q.E.D.

REMARK. In case $f$ generates a finite cyclic group $H$, the relation between the above theorem and [7] can be illustrated locally as follows: Let a finite 
group $H \times G$ act on a $C^{\infty}$-manifold $M$ preserving the orientation. Then it induces an orientation-preserving $H$-action on the $V$-manifold $M / G$. Let $\tilde{A}$ be a $H \times G$-invariant elliptic operator on $M$, then it induces an $H$-invariant elliptic operator $A$ on $M / G$ [1]. The index of $A$ on $M / G$ can be defined as the index of the operator $\tilde{A}$ over $M$ restricted to the space of $G$-invariant sections. Then it follows from the theorem in $[5$, p. 76] that for $f \in H$, ind $_{M / G}(A)(f)=|G|^{-1} \Sigma_{g \in G} \operatorname{ind}_{M}(\tilde{A})(f g)$.

The author wishes to thank Tyrone Duncan for helpful conversations.

\section{REFERENCES}

1. M. F. Atiyah, Elliptic operators and compact groups, Lecture Notes in Math., vol. 401, Springer-Verlag, Berlin-Heidelberg-New York, 1974.

2. M. F. Atiyah, R. Bott, and V. K. Padoti, On heat equation and index theorem, Invent. Math. 19 (1973), 279-330.

3. M. F. Atiyah and I. M. Singer, The index of elliptic operators. III, Ann. of Math. (2) 87 (1968), 546-604.

4. W. L. Baily, The decomposition theorem for $V$-manifolds, Amer. J. Math. 78 (1956), 862-888.

5. F. Hirzebruch and D. Zagier, The Atiyah-Singer theorem and elementary number theory, Publish or Perish Inc., Boston, Mass., 1974.

6. T. Kawasaki, An analytic Lefschetz fixed point formula and its application to $V$-manifolds: general defect formula and Hirzerbruch signature theorem, $\mathrm{Ph} . \mathrm{D}$. Thesis, Johns Hopkins Univ., 1976.

7. T. Kawasaki, The signature theorem for $V$-manifolds, Topology 17 (1978), 75-83.

8. I. Satake, On a generalization of the notion of manifold, Proc. Nat. Acad. Sci. U.S.A. 42 (1956), 359-365.

9. __ The Gauss-Bonnet theorem for V-manifolds, J. Math. Soc. Japan 9 (1957), 464-492.

Department of MAThematics, UNiversity OF KANSAS, LAWREnCe, Kansas 66045 